\title{
An adaptive compound control system for the ESC of electric-wheel vehicle
}

\author{
Cheng Wang ${ }^{1}$, Mingxiang Tang ${ }^{1}$, Chuanxue Song ${ }^{1, a}$, Jianhua $\mathrm{Li}^{1}$ \\ 1 Jilin University, China
}

\begin{abstract}
The aim of this study is to achieve the adaptive control for the Electronic Stability Control (ESC) of electric-wheel vehicle. An adaptive compound control system is designed. The system main includes a yaw velocity controller and a side slip angle controller. The yaw velocity controller is robust PID. The side slip angle controller is neural network PID. The PID parameters are adjusted adaptively through robust and neural network. The two controllers constitute the compound controller. Lateral acceleration is used as a limit value and added to the yaw velocity control. A full vehicle model is built to simulate the real electric-wheel vehicle. The ideal values of control parameters are introduced through the ideal vehicle model. Simulation experiments were dong, which included a steering wheel step input experiment and a sine input experiment. The experimental results show that the steady state and the transient performance of the control system are good. The adaptive compound control system is fit for the ESC.
\end{abstract}

\section{Introduction}

The electric-wheel vehicle cancels the traditional power transmission devices. The wheel hub motors drive the electric-wheel vehicle independently. ESC can ensure the vehicle driving stability. Improvement of the ESC performance is becoming a research hotspot. The yaw moment control is the main task of the ESC. The characteristic parameters of the yaw moment control are the yaw velocity and the side slip angle. The conventional ESC system adopts Proportion Integration Differentiation (PID) controller to control these parameters. PID controller lacks adaptive adjusting parameter ability online. This makes PID can not well adapt to the complex road conditions [1].

\section{Adaptive compound ESC system structure}

The structure of the adaptive compound ESC system is as Fig. 1. The ideal vehicle model outputs the ideal values. The full vehicle model outputs the actual values. The state estimator outputs the estimated value. The control parameters are the yaw velocity and the side slip angle. The yaw velocity controller is robust PID. It controls the error of the yaw velocity. The side slip angle controller is neural network PID. It controls the error of the side slip angle. The control model discrimination controls the working state of the controllers. A threshold of the side slip angle decides work and stop of the two controllers. The yaw moment distribution calculates the moment distribution coefficient. The wheel hub motor controller is used to control the yaw moment of four wheels [2].

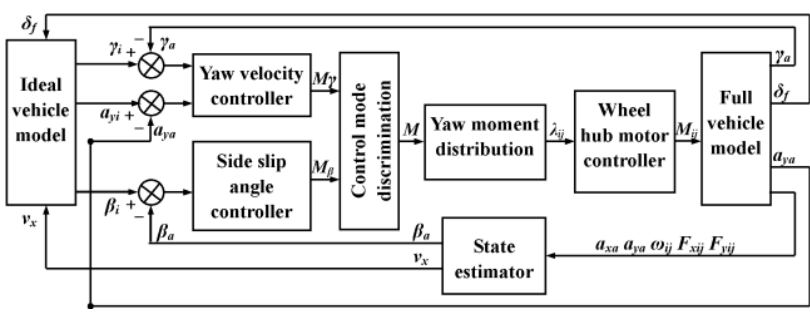

Figure 1. Adaptive compound ESC system structure

\section{Electric-wheel vehicle dynamics modeling}

\subsection{Full vehicle model}

The full vehicle model includes a vehicle model and a tire model. The tire model adopts Dugoff tire model [3]. The vehicle model is eight degrees of freedom. It is as Fig. 2. The model includes longitudinal movement, lateral movement, yaw movement, four wheels rotation and front wheel steering [4].

The longitudinal movement and the lateral movement are as follows:

$$
\left\{\begin{aligned}
& m a_{x}=m\left(\dot{v}_{x}-v_{y} \gamma\right) \\
& m\left(\dot{v}_{x}-v_{y} \gamma\right)=\left(F_{x f l}+F_{x f r}\right) \cos \delta_{f}-\left(F_{y f l}+F_{y f r}\right) \sin \delta_{f} \\
&+F_{x r l}+F_{x r r}
\end{aligned}\right.
$$

\footnotetext{
a Corresponding author: lwtg0915wang@126.com
} 


$$
\left\{\begin{aligned}
& m a_{y}=m\left(\dot{v}_{y}+v_{x} \gamma\right) \\
& m\left(\dot{v}_{y}+v_{x} \gamma\right)=\left(F_{x f l}+F_{x f r}\right) \sin \delta_{f}+\left(F_{y f l}+F_{y f r}\right) \cos \delta_{f} \\
&+F_{y r l}+F_{y r r}
\end{aligned}\right.
$$

$v_{x}$ is the longitudinal speed. $v_{y}$ is the lateral speed. $a_{x}$ is the longitudinal acceleration. $a_{y}$ is the lateral acceleration. $Y$ is the yaw velocity. $\delta_{f}$ is the front wheel angle. $m$ is the vehicle mass. $F_{x i j}$ is the longitudinal force. $F_{y i j}$ is the lateral force.

The yaw movement is as follow:

$$
\begin{aligned}
I_{z} \dot{\gamma}= & -l_{f}\left[\sin \delta_{f}\left(F_{x f l}+F_{x f r}\right)+\cos \delta_{f}\left(F_{y f l}+F_{y f r}\right)\right] \\
& +\frac{l_{a}}{2}\left[\cos \delta_{f}\left(F_{x f r}-F_{x f l}\right)+\sin \delta_{f}\left(F_{y f l}-F_{y f r}\right)\right] \\
& +l_{r}\left(F_{y r l}+F_{y r r}\right)+\frac{l_{b}}{2}\left(F_{x r r}-F_{x r l}\right)
\end{aligned}
$$

$I_{z}$ is the rotational inertia around $\mathrm{z}$ axis. $I_{f}$ is the front wheel base. $I_{r}$ is the rear wheel base.

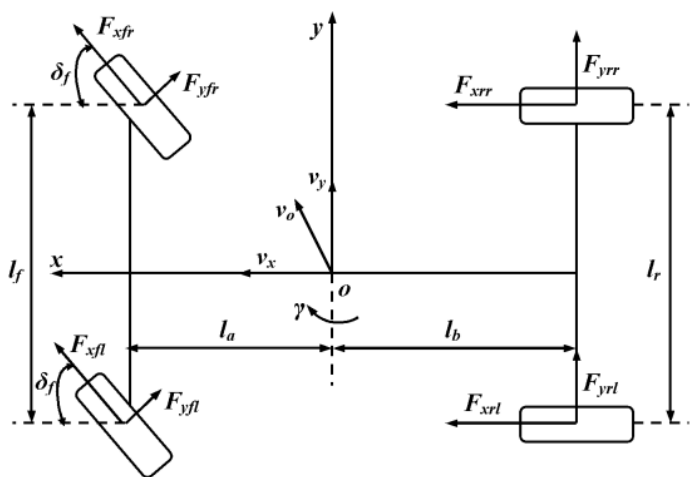

Figure 2. Eight degrees of freedom vehicle model

The four wheels rotation is as follow:

$$
I_{i j} \dot{\omega}_{i j}=\left(T_{m} / i_{m}-F_{x i j} R_{w}-f_{w} F_{z i j}\right)
$$

$I_{i j}$ is the wheel rotational inertia. $\omega_{i j}$ is the wheel angular speed.

The front wheel steering is as follow:

$$
I_{f} \ddot{\delta}_{f}+c_{s} \dot{\delta}_{f}+i_{s}^{2} k_{s} \delta_{f}=i_{s} k_{s} \delta_{s w}+k_{f} \alpha_{f}-t_{m} F_{y f}
$$

$\delta_{s w}$ is the steering wheel angle. $\alpha_{f}$ is the side slip angle of the front wheel.

\subsection{Ideal vehicle model}

The ideal vehicle model is two degree of freedom. It includes the lateral movement and the yaw movement. It is as follows [5]:

$$
\begin{gathered}
m a_{y}=\left(c_{f}+c_{r}\right) \beta+\frac{\left(l_{a} c_{f}-l_{b} c_{r}\right)}{v_{x}} \gamma-c_{f} \delta_{f} \\
I_{z} \dot{\gamma}=\left(l_{a} c_{f}-l_{b} c_{r}\right) \beta+\frac{\left(l_{a}^{2} c_{f}+l_{b}^{2} c_{r}\right)}{v_{x}} \gamma-l_{a} c_{f} \delta_{f}
\end{gathered}
$$

$\beta$ is the side slip angle. $c_{f}$ is the lateral stiffness of the front wheel. $c_{r}$ is the lateral stiffness of the rear wheel.

After the ideal vehicle model is carried on the Laplace transform, the gain of the yaw velocity and the side slip angle can be calculated [6]. The gain of the yaw velocity is as follow:

$$
G_{\gamma}=\frac{v_{x}}{L\left(1+\xi v_{x}^{2}\right)}
$$

$G_{Y}$ is the gain of the yaw velocity.

The gain of the side slip angle is as follow:

$$
G_{\beta}=\frac{l_{b}-\frac{m l_{a} v_{x}^{2}}{c_{r} L}}{L\left(1+\xi v_{x}^{2}\right)}
$$

$\xi$ is the stability factor. $L=l_{a}+l_{b}$.

The steady state value of the yaw velocity is as follow:

$$
\gamma_{s}=\frac{v_{x}}{L\left(1+\xi v_{x}^{2}\right)} \delta_{f}
$$

The steady state value of the side slip angle is as follow:

$$
\beta_{s}=\frac{l_{b}-\frac{m l_{a} v_{x}^{2}}{c_{r} L}}{L\left(1+\xi v_{x}^{2}\right)} \delta_{f}
$$

According to the actual situation of the road, the limit values of the yaw velocity and the side slip angle are as follows:

$$
\begin{gathered}
\gamma_{l}=0.85 \frac{\mu g}{v_{x}} \\
\beta_{l}=\tan ^{-1}(0.02 \mu g)
\end{gathered}
$$

Considering the steady state values and the limit values at the same time, the ideal value of the yaw velocity is as follow:

$$
\gamma_{i}=\min \left\{\left|\frac{v_{x}}{L\left(1+\xi v_{x}^{2}\right)}\right| \delta_{f},\left|0.85 \frac{\mu g}{v_{x}}\right|\right\} \operatorname{sgn}\left(\delta_{f}\right)
$$

$Y_{i}$ is the ideal value of the yaw velocity.

The ideal value of the side slip angle is as follow:

$$
\beta_{i}=\min \left\{\left|\frac{l_{b}-\frac{m l_{a} v_{x}^{2}}{c_{r} L}}{L\left(1+\xi v_{x}^{2}\right)}\right| \delta_{f},\left|\tan ^{-1}(0.02 \mu g)\right|\right\} \operatorname{sgn}\left(\delta_{f}\right)
$$

$\beta_{i}$ is the ideal value of the side slip angle. $\mu$ is the road adhesion coefficient. 


\section{Adaptive controller design}

\subsection{Adaptive yaw velocity controller}

The adaptive yaw velocity controller is as Fig. 3. It includes two robust PID. One is for the yaw velocity control. The other is for the lateral acceleration control. The lateral acceleration control is a restriction in the controller.

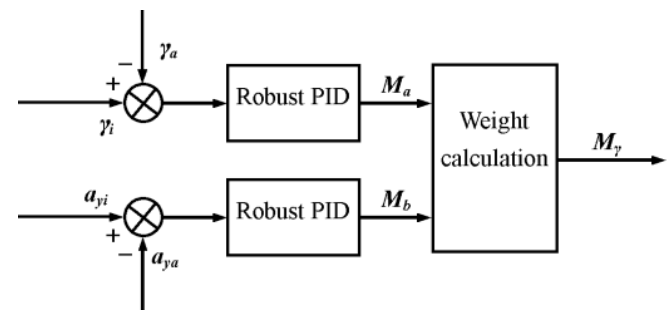

Figure 3. Adaptive yaw velocity controller

Based on the theory of $\mathrm{H}_{\infty}$, the transfer function of robust PID is as follow [7]:

$$
G(s)=2 \frac{T}{K}\left(1+\frac{1}{T_{I} s}\right) \frac{T_{D} s+1}{2 \tau^{2} t^{2}+4 \tau t+t}
$$

$T$ is the time constant. $T_{I}$ is the integral time constant. $T_{D}$ is the differentiating time constant. $t$ is the delay time. $T$ is the impact factor of PID.

The impact factor changes the control parameters of the robust PID.

The rule of the impact factor is as follow:

$$
\begin{cases}0<e<e_{1} & \tau=\tau_{1} \\ e_{1} \leq e<e_{2} & \tau=\tau_{2} \\ e_{2} \leq e & \tau=\tau_{3}\end{cases}
$$

$e$ is the error of the ideal value and the actual value. $e_{1}$ and $e_{2}$ are the threshold.

The ideal lateral acceleration is based on the neutral steering. The neutral steering is as follow:

$$
a_{y m}=\frac{v_{x}^{2}}{L} \delta_{f}+\dot{v}_{y}
$$

The ideal lateral acceleration is as follow:

$$
\varepsilon_{1} a_{y m} \leq a_{y i} \leq \varepsilon_{2} a_{y m}
$$

$a_{y i}$ is the ideal lateral acceleration. $a_{y m}$ is the neutral steering value. $\varepsilon_{1}$ and $\varepsilon_{2}$ are the range factor.

The moment $M_{a}$ can be obtained by control the error of the yaw velocity. The moment $M_{b}$ can be obtained by control the error of the lateral acceleration [8].

The control law of the adaptive yaw velocity controller is as follows:

$$
\begin{aligned}
M_{a}(k)= & K_{p} \Delta e_{\gamma}(k)+K_{I} e_{\gamma}(k)+K_{D} \frac{d e_{\gamma}(k)}{d t} \\
& +M_{a}(k-1)
\end{aligned}
$$

$$
\begin{gathered}
M_{b}(k)=K_{p} \Delta e_{a y}(k)+K_{I} e_{a y}(k)+K_{D} \frac{d e_{a y}(k)}{d t} \\
+M_{b}(k-1) \\
M_{y}=\lambda M_{a}+(1-\lambda) M_{b}
\end{gathered}
$$

$\lambda$ is the weight, it is adjusted by the weight calculation. $M_{Y}$ is the total moment.

\subsection{Adaptive side slip angle controller}

The adaptive side slip angle controller is as Fig. 4.

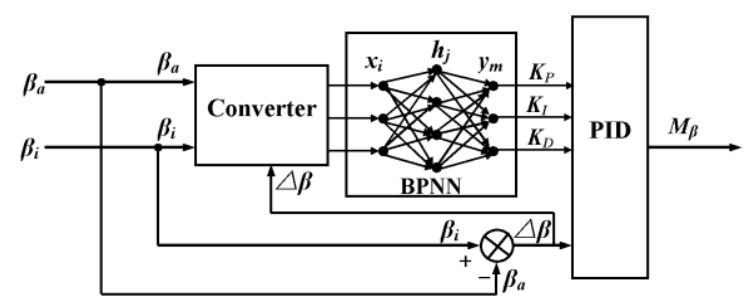

Figure 4. Adaptive side slip angle controller

Back Propagation Neural Network (BPNN) includes the input layer, the hidden layer and the output layer. The learning process of BPNN is the forward propagation and the reverse propagation [9]. The side slip angle controller is built based on an improved BPNN.

In the improved BPNN, two position actors are added to the activation function. The factors are $\sigma$ and $\rho$. The factors are used to expand the weight range and speed up the learning speed.

The activation function is as follow:

$$
f(x)=\frac{e^{2(x-\sigma)}-1}{e^{2(x-\sigma)}+1}+\rho
$$

The input of the improved BPNN is the ideal value, the actual value and the error of the side slip angle. The output is the proportionality coefficient, the integral coefficient and the differential coefficient of PID.

The input is as follows:

$$
\begin{aligned}
& \left\{\begin{array}{l}
x_{1}=\beta_{i} \\
x_{2}=e_{\beta} \\
x_{3}=\beta_{a}
\end{array}\right. \\
& e_{\beta}=\beta_{i}-\beta_{a}
\end{aligned}
$$

The output is as follow:

$$
\left\{\begin{array}{l}
y_{1}=K_{P} \\
y_{2}=K_{I} \\
y_{3}=K_{D}
\end{array}\right.
$$

The calculation process of the hidden layer is as follow:

$$
h_{j}=f\left(\sum_{i=1}^{3} v_{i j} x_{i}\right) \quad j=1,2,3,4
$$


$x_{i}$ is the input of the input layer. $h_{j}$ is the nerve cell output of the hidden layer. $v_{i j}$ is the weight from the input layer to the hidden layer.

The calculation process of the input layer is as follow:

$$
y_{m}=f\left(\sum_{j=1}^{4} w_{j m} h_{j}\right) \quad m=1,2,3
$$

$y_{m}$ is the output of the output layer. $w_{j m}$ is the weight from the hidden layer to the output layer.

The update of the weights is as follows [10]:

$$
\begin{gathered}
w_{j m}(k+1)=w_{j m}(k)+\eta \delta_{m} h_{j} \\
+\psi\left[w_{j m}(k)-w_{j m}(k-1)\right] \\
v_{i j}(k+1)=v_{i j}(k)+\eta \delta_{j} x_{i}+\psi\left[v_{i j}(k)-v_{i j}(k-1)\right]
\end{gathered}
$$

$\eta$ is the learning rate. $\psi$ is the damping factor. $\delta$ is the back propagation error.

The control law of the adaptive side slip angle controller is as follow:

$$
\begin{aligned}
M_{\beta}(k)= & K_{P} \Delta e_{\beta}(k)+K_{I} e_{\beta}(k) \\
& +K_{D} \frac{d e_{\beta}(k)}{d t}+M_{\beta}(k-1)
\end{aligned}
$$

\section{Simulation experiment}

The simulation experiments were based on the combined simulation of AMEsim and Simulink. The experimental purpose is to verify the effect of the designed control system on the ESC control parameters.

The experiments included the step input experiment and the sine input experiment.
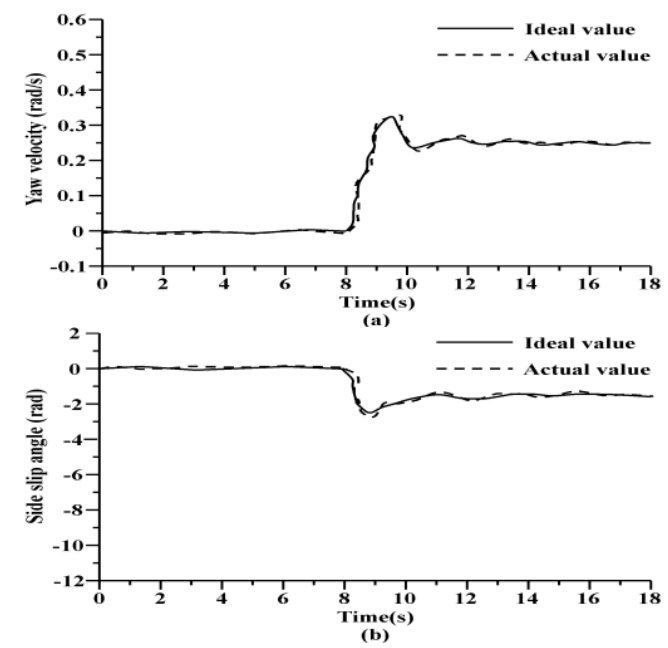

Figure 5. Experimental results of step input

In the step input experiment, the vehicle speed was $100 \mathrm{~km} / \mathrm{h}$; the step angle was $0.25 \mathrm{rad}$. The experimental results are as Fig. 5.

In the sine input experiment, the vehicle speed was 20 $\mathrm{km} / \mathrm{h}$; the steering wheel rotated according to sine. The experimental results are as Fig. 6.
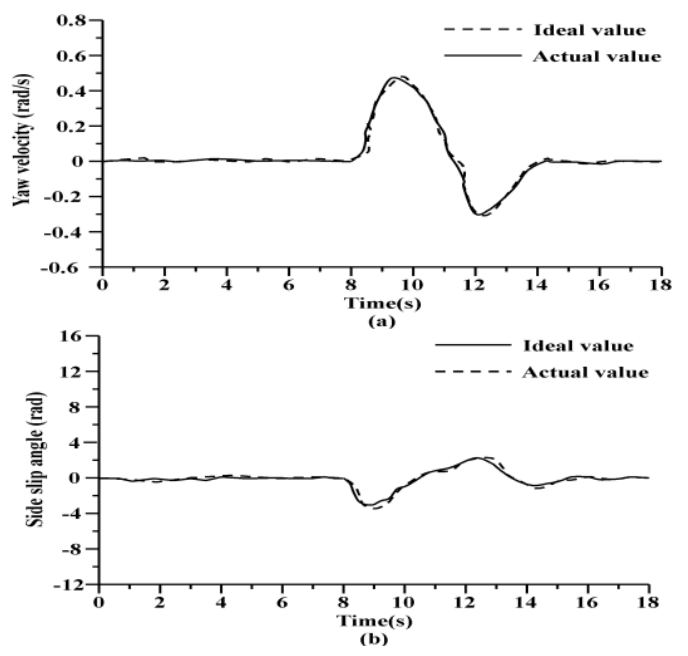

Figure 6. Experimental results of sine input

It can be seen from the experimental results that the actual values can track the ideal values well. The response of the adaptive compound control system is timely and accurate. The maximum overshoot and the delay time of the system can satisfy the requirements of actual control.

\section{Conclusion}

The electric-wheel vehicle dynamic model helps to simulate the real vehicle and calculate the ideal values of control parameters. The robust PID can enhance the robustness of the yaw velocity control. The neural network PID can improve the timeliness of the side slip angle control. The PID parameters can be adjusted adaptively online. From the experimental results, the steady state and the transient performance of the control system are good. The adaptive compound control system is fit for the ESC of electric-wheel vehicle.

\section{References}

1. L. Wang, L. Tan, L. An, Z. Wu, L. Li, J. Electr. Syst 8, 57 (2012)

2. D. Yin, J.S. Hu, Int. J. Automot. Technol 15, 979 (2014)

3. H. Wang, Z. Shi, Chin. J. Control. Theory. Appl 32, 837 (2015)

4. S. Cui, Vehicle system dynamics and simulation, Peking University Press, Peking (2014)

5. Z. Yu, Automobile theory, China Machine Press, Peking (2009)

6. M.J. Elzinga, J. R. Soc. Interface 9, 1685 (2012)

7. G.E. Dullerud, A course in robust control theory, World Book Inc, Peking (2014)

8. D. Jiang, D. Li, S. Wang, L. Tian, L. Yang, Int. J. Adv. Rob. Syst 12 (2015)

9. S. Haykin, Neural networks and learning machines, China Machine Press, Peking (2009)

10. J. Zheng, M. Xiao, M. Yang, Y. Li, L. Kong, Int. J. Online Eng 9, 40 (2013) 